\title{
SMOOTH HOMOTOPY PROJECTIVE SPACES
}

\author{
BY CHARLES H. GIFFEN 1
}

\author{
Communicated by William Browder, September 19, 1968
}

Introduction. In [5] we considered certain fixed point free involutions on Brieskorn manifolds as weakly complex bordism elements. In [4] we considered associated examples of smooth normal invariants for real projective spaces, settling the realizability question for dimensions $\not \equiv 1 \bmod 4$ and the desuspendability question for dimensions $4 k+1$. The object of this study is the classification of these smooth normal invariants given by the Brieskorn examples. Our results overlap somewhat with Atiyah and Bott [2] as well as Browder [3], but our methods are entirely different and our results rather more refined. Full details of these and related results will appear elsewhere.

1. Smooth normal invariants. Following Sullivan [6], we regard a smooth normal invariant of a space $X$ as an element of $[X, G / O]$. Of course, we have $G / O \cong S G / S O$. We need the fibers $S G /$ Spin of $B$ Spin $\rightarrow B S G$ and $S O /$ Spin $\simeq P^{\infty}$ of $B$ Spin $\rightarrow B S O$. The spaces $S G / S O$, $S G /$ Spin, $S O /$ Spin have their Whitney $H$-space structures under which the sequence

$$
S O / \text { Spin } \rightarrow S G / \text { Spin } \rightarrow S G / S O
$$

is a multiplicative fibration.

A map $\mu: S G /$ Spin $\rightarrow B O$ is constructed as follows. Let $\gamma_{n}$ denote the universal fiber space over $B S G_{n}$ with fiber $S^{n-1}, \beta_{n}$ the pullback to $B S \operatorname{pin}_{n}$, and $\alpha_{n}$ the pullback to $S G_{n} / \mathrm{Spin}_{n}$; also, let $\epsilon_{n}$ denote the $S^{n-1}$ fibration over a point. Corresponding to the commutative diagram

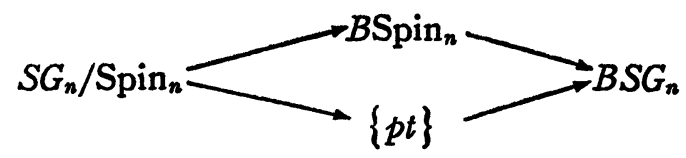

of spaces, there is the commutative diagram

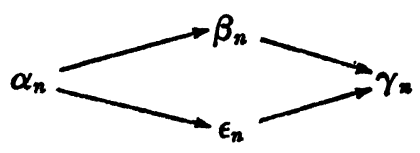

${ }^{1}$ Research supported by NSF grant GP-6567. 
of induced $S^{n-1}$ fibrations. Passing to Thom spaces we obtain a commutative diagram of spectra

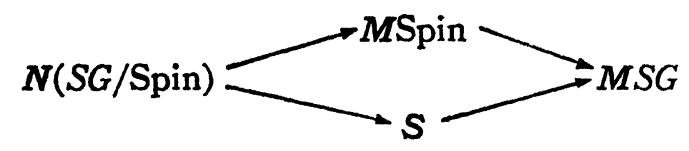

where $S$ is the sphere spectrum and $N(S G /$ Spin) is the spectrum with $N(S G / \text { Spin })_{n}=T\left(\alpha_{n}\right) \simeq S^{n} \wedge\left(S G_{n} / \operatorname{Spin}_{n}\right)^{+}$. There is a map of spectra $M$ Spin $\rightarrow b O$, where $b O$ is the $\Omega$-spectrum with $b O_{0}=\boldsymbol{Z} \times B O$, defining the $k O$-orientation of Spin cobordism. Now the composition

$$
N(S G / \text { Spin }) \rightarrow M \text { Spin } \rightarrow b O
$$

of maps of ring spectra defines $\mu: S G / \operatorname{Spin} \rightarrow B O$ in the usual way, since $N(S G /$ Spin $)$ and $S \wedge(S G / \text { Spin })^{+}$are equivalent.

(1.1) Theorem. The map $\mu: S G / \mathrm{Spin} \rightarrow B O$ is an $H$-map from the Whitney structure of $S G /$ Spin to the tensor product structure of $B O$.

The following is a central fact in our study.

(1.2) TheOREM. The composition

$$
P^{\infty} \simeq S O / \text { Spin } \rightarrow S G / \text { Spin } \rightarrow B O
$$

classifies the canonical line bundle $\eta$ over $P^{\infty}$.

Since this map $\eta: P^{\infty} \rightarrow B O$ splits the map $w_{1}: B O \rightarrow K\left(Z_{2}, 1\right) \simeq P^{\infty}$, we get the following easily.

(1.3) Corollary. The above maps fit into a commutative diagram

$$
\begin{array}{ccr}
P^{\infty} & \rightarrow S G / \text { Spin } & \rightarrow G / O \\
\| & \downarrow \mu & \downarrow \nu \\
P^{\infty} \stackrel{\eta}{\rightarrow} \quad B O \rightarrow B S O
\end{array}
$$

of maps of $H$-spaces (BO and BSO with the tensor product structure), each row being a multiplicative fibration.

Using Poincaré duality for Spin cobordism theory, we are able to make explicit computations of the $k O$-orientation $\mu: S G / \mathrm{Spin} \rightarrow B O$. This amounts to interpreting $N(S G /$ Spin $) \rightarrow M$ Spin in terms of Spin bordism of Spin manifolds.

For a finite $C W$ complex $X$, we take a homotopy equivalent compact Spin manifold $M^{m}$. An element of $\left[M^{m}, S G /\right.$ Spin] is represented by a spherical fiber bundle over $M^{m}$ with Spin structural group and an $S G$ (i.e. degree +1 fiber homotopy) trivialization. Using transverse 
regularity on the $S G$ trivialization, we obtain an element $\left[V^{m}, \partial V^{m} ; e\right]$ $\in \Omega_{m}^{\mathrm{Spin}}\left(M^{m}, \partial M^{m}\right)$ of degree +1 ( $e$ is the bundle projection). The Poincaré duality isomorphism sends this to an element of $\Omega_{\text {Spin }}^{0}\left(M^{m}\right)$ with augmentation +1 . Now applying the $k O$-orientation of Spin cobordism, we obtain a virtual line bundle over $M^{m}$, i.e. an element of $\left[M^{m}, B O\right]$. This describes the natural map $[X, \mu]:[X, S G /$ Spin $]$ $\rightarrow[X, B O]$.

2. Spin cobordism of projective spaces. At this point the Brieskorn examples are brought into play. The degree $q$ maps $h: Q_{q}^{4 k-1} \rightarrow P^{4 k-1}$ of $[5,83]$ may be taken to represent elements $\left[Q_{a}^{4 k-1}, h\right] \in \Omega_{4 k-1}^{\text {Spin }}\left(P^{4 k-1}\right)$ in a canonical way so that the following analogue of [5, Theorem 3.4] holds.

(2.1) Theorem. The Poincaré duals $b_{q}^{4 k-1} \in \Omega_{\mathrm{Spin}}^{0}\left(P^{4 k-1}\right)$ of the elements $\left[Q_{a}^{4 k-1}, h\right] \in \Omega_{4 k-1}^{\mathrm{Spin}}\left(P^{4 k-1}\right)$ satisfy $b_{a}^{4 k-1}=q \cdot 1$.

It happens that $h: Q_{1}^{4 k-1} \rightarrow P^{4 k-1}$ is a diffeomorphism, making it clear what Spin structures to use. Actually, the above holds equally well for $S p$ and $S U$ cobordism of $P^{4 k-1}$.

To see what happens for other dimensions, just cut the maps $h: Q_{a}^{4 k-1} \rightarrow P^{4 k-1}$ down to a suitably small smooth regular neighborhood of $P^{n} \subset P^{4 k-1}$, obtaining $h: V_{q}^{4 k-1, n} \rightarrow M^{4 k-1, n}$, say. Note that the Brieskorn examples $Q_{e}^{n}$ of [4] are the transverse regular inverse images in $Q_{a}^{4 k-1}$ of $P^{n} \subset P^{4 k-1}$. The resulting elements $b_{a}^{n} \in \Omega_{\text {Spin }}^{0}\left(P^{n}\right)$ are just the inclusion induced pullbacks of the elements $b_{a}^{4 k-1}$ for $n \leqq 4 k-1$.

(2.2) Corollary. In $\Omega_{\mathrm{Spin}}^{0}\left(P^{n}\right)$ we have $b_{q}^{n}=q \cdot 1$.

The double covering $e: S^{4 k-1} \rightarrow P^{4 k-1}$ defines the element $\left[S^{4 k-1}, e\right]$ $\in \Omega_{4 k-1}^{\text {Spin }}\left(P^{4 k-1}\right)$ of degree +2 . For each $n$, Poincaré duality and pulling back from $P^{4 k-1}$ to $P^{n}$ produce the elements $s^{n} \in \Omega_{\text {Spin }}^{0}\left(P^{n}\right)$ with augmentation +2 . To emphasize the fact that $s^{n} \neq 2 \cdot 1$ we point out the following.

(2.3) TheOREM. Under the kO-orientation morphism

$$
\Omega_{\mathrm{Spin}}^{*}\left(P^{n}\right) \rightarrow k O^{*}\left(P^{n}\right)
$$

$s^{m \rightarrow 1+\eta}$, where $\eta$ is the canonical line bundle.

The following, on the other hand, is straightforward geometry.

(2.4) Proposition. For any integer $j$ there is a map

$$
h^{\prime}:\left(V_{q}^{4 k-1, n}, \partial V_{q}^{4 k-1, n}\right) \rightarrow\left(M^{4 k-1, n}, \partial M^{4 k-1, n}\right)
$$


of degree $q+2 j$ such that the Poincare dual of $\left[V_{a}^{4 k-1, n}, \partial V_{a}^{4 t-1, n} ; h^{\prime}\right]$ is $b_{a}^{n}+j \cdot s^{n} \in \Omega_{\text {Spin }}^{0}\left(P^{n}\right)$.

3. The Brieskorn normal invariants. In [4] we pointed out how the Brieskorn examples $Q_{2 d+1}^{n}$ produce smooth normal invariants of $P^{n}$. Here we show how these examples lead to elements of $\left[P^{n}, S G / \mathrm{Spin}\right]$ compatible with the machinery of the previous section.

As remarked in [4], each $Q_{2 d+1}^{4 k+1}$ is homotopy equivalent to $P^{4 k+1}$. Consequently the map

$$
h_{2 d+1}:\left(V_{2 d+1}^{4 k+3,4 k+1}, \partial V_{2 d+1}^{4 k+3,4 k+1}\right) \rightarrow\left(M^{4 k+3,4 k+1}, \partial M^{4 k+3,4 k+1}\right)
$$

of degree +1 provided by (2.4) is a homotopy equivalence of Spin manifolds. Now the construction of [6] produces in our case a "classifying" $S G /$ Spin-bundle over $M^{4 k+3,4 k+1} \sim P^{4 k+1}$ for $h_{2 d+1}$, i.e. an element $a_{2 d+1}^{4 k+1} \in\left[P^{4 k+1}, S G /\right.$ Spin $]$. Restriction to $P^{n} \subset P^{4 k+1}$ defines the element $a_{2 d+1}^{n} \in\left[P^{n}, S G / \mathrm{Spin}\right]$.

Now we follow the description of $\left[P^{n}, \mu\right]$ as given in $\$ 1$. We find that $a_{2 d+1}^{n}$ leads to the element $b_{2 d+1}^{n}-d \cdot s^{n} \in \Omega_{\text {Spin }}^{0}\left(P^{n}\right)$ which by (2.2) and $(2.3)$ is sent (under $k O$-orientation) to the element $1+d \cdot \xi$ $\in k O^{0}\left(P^{n}\right)$, where $\xi=1-\eta$. This gives our main result on the Brieskorn examples.

(3.1) THEOREM. Under kO-orientation we have

$$
\left[P^{n}, \mu\right]\left(a_{2 d+1}^{n}\right)=1+d \cdot \xi
$$

as a virtual line bundle over $P^{n}$.

Numerous results follow from (3.1), in particular the following which stems from (1.3).

(3.2) ThEоRем. The natural maps

$$
\begin{aligned}
& {\left[P^{n}, \mu\right]:\left[P^{n}, S G / \text { Spin }\right] \rightarrow\left[P^{n}, B O\right],} \\
& {\left[P^{n}, \nu\right]:\left[P^{n}, G / O\right] \rightarrow\left[P^{n}, B S O\right],}
\end{aligned}
$$

are epimorphisms of groups (where BO and BSO have their tensor product structures).

Results of Browder [3] can be applied to show that the epimorphisms in (3.2) are canonically split-in fact, that $\left[P^{n}, \nu\right]$ classifies the Brieskorn examples of smooth normal invariants for $P^{n}$. Similarly, $\left[P^{n}, \mu\right]$ classifies the Brieskorn elements $a_{2 d+1}^{n} \in\left[P^{n}, S G / \mathrm{Spin}\right]$. Browder's results alone do not give this except for $n \leqq 5$.

4. Numerical results. By results of Adams [1], $k O^{0}\left(P^{n}\right)=K O\left(P^{n}\right)$ 
is the commutative ring generated by 1 and $\xi=1-\eta$ subject to the relations $\xi^{2}=2 \cdot \xi$ and $a_{n+1} \cdot \xi=0$, where $a_{k}$ is given by the table:

\begin{tabular}{|c|c|c|c|c|c|c|c|c|}
\hline$k$ & 1 & 2 & 3 & 4 & 5 & 6 & 7 & $8 \cdots j+8$ \\
\hline$a_{k}$ & 1 & 2 & 4 & 4 & 8 & 8 & & $8 \cdots 16 a_{j}$ \\
\hline
\end{tabular}

Now it is clear that the multiplicative group of virtual line bundles $1+d \cdot \xi \in\left[P^{n}, B O\right]$ is isomorphic to $Z_{2} \times Z_{a_{n+1} / 2 .}$. Moreover, the two generators $1-\xi=\eta$ and $1-2 \cdot \xi=2 \cdot \eta-1$ of $\left[P^{n}, B O\right]$ generate the $Z_{2}$ and $Z_{a_{n+1} / 2}$ factors corresponding to $\left[P^{n}, P^{\infty}\right]$ and $\left[P^{n}, B S O\right]$, respectively-as indicated in the following diagram:

$$
\begin{array}{cc}
{\left[P^{n}, P^{\infty}\right]} & \rightleftarrows\left[P^{n}, B O\right] \rightleftarrows\left[P^{n}, B S O\right] \\
\mathbb{R} & \mathbb{R} \quad \\
Z_{2} & \rightleftarrows Z_{2} \times Z_{a_{n+1} / 2} \rightleftarrows Z_{a_{n+1} / 2}
\end{array}
$$

(4.1) Theorem. There are $a_{n+1} / 2$ distinct Brieskorn smooth normal invariants of $P^{n}$.

(4.2) CoRollary. There are $a_{4 k+2} / 2=2^{2 k}$ smoothly distinct Brieskorn homotopy projective $(4 k+1)$-spaces. For $k>0$, these yield only 4 combinatorially distinct homotopy projective $(4 k+1)$-spaces.

(4.3) CoRollaRy. For $n \neq 1 \bmod 4$ and $n>5$, there are $a_{n+1} / 4$ smoothly distinct homotopy projective $n$-spaces which yield only 2 combinatorially distinct homotopy projective $n$-spaces.

\section{REFERENCES}

1. J. F. Adams, Vector fields on spheres, Ann. of Math. (2) 75 (1962), 603-632.

2. M. F. Atiyah and R. Bott, A Lefschetz fixed point formula for elliptic complexes. II, Ann. of Math. (2) 88 (1968), 451-491.

3. William Browder (to appear).

4. C. H. Giffen, Desuspendability of free involutions on Brieskorn spheres, Bull. Amer. Math. Soc. 75 (1969), 426-429.

5. - Weakly complex involutions and cobordism of projective spaces (to appear).

6. D. P. Sullivan, Triangulating and smoothing homotopy equivalences and homeomorphisms, Geometric Topology Seminar Notes (mimeographed), Princeton University, Princeton, N. J., 1967.

University of Virginia, Charlottesville, Virginia 22901 